\title{
Human Periodontal Ligament Characterization by Means of Vibrational Spectroscopy and Electron Microscopy
}

\author{
Carlo Camerlingo 1, Fabrizia d'Apuzzo 2, Marcella Cammarota ${ }^{3}$, Sonia Errico ${ }^{3}$, Marianna \\ Portaccio $^{3}$, Letizia Perillo ${ }^{2}$ and Maria Lepore ${ }^{3, *}$ \\ 1 CNR-SPIN, Istituto Superconduttori, Materiali Innovativi e Dispositivi, via Campi Flegrei 34, Pozzuoli \\ 80078, Italy \\ 2 Dip. Multidisciplinare di Specialità Medico-Chirurgiche e Odontoiatriche, Università degli Studi della \\ Campania Luigi Vanvitelli, via L. De Crecchio 6, Napoli 80138, Italy \\ 3 Dip. di Medicina Sperimentale, Università degli Studi della Campania Luigi Vanvitelli, via S. Maria di \\ Costantinopoli 16, Napoli 80138, Italy \\ * Correspondence: maria.lepore@unicampania.it \\ + Presented at the 7th Electronic Conference on Sensors and Applications, 15-30 November 2020; Available \\ online: https://ecsa-7.sciforum.net/.
}

Published: 15 November 2020

\begin{abstract}
Human periodontal ligament (PDL) is a membrane-like connective tissue interposed between the tooth root and the alveolar bone of which the main component is represented by collagen fibers. During the early stage of application of orthodontic forces different changes occur in PDL. For this reason, its characterization with conventional and non-conventional techniques can be extremely interesting. We investigated samples of PDL of orthodontic patients, aged between 13 and 21 years, using different experimental techniques. Morphological characterization of PDL samples was carried out by using a scanning electron microscope. Fourier Transform Infrared $(\mu-$ FT-IR) and Raman ( $\mu$-RS) microspectroscopies were used for biochemical characterization of PDL samples. A biochemical characterization of PDL tissues with clear evidence of contributions from collagen, lipid and other protein was obtained. The analysis of Amide I and Amide III components was also performed giving indication on the protein secondary structure.
\end{abstract}

Keywords: Periodontal ligament; gingival crevicular fluid; Raman microspectroscopy; FourierTransform infrared microspectroscopy

\section{Introduction}

The periodontal ligament (PDL) is the structure interposed between the tooth root and the alveolar bone, occupying an area of 0.25 to $0.5 \mathrm{~mm}^{2}$. It is a connective tissue whose main component is represented by a set of collagen elastic fibers, parallel to each other, which are inserted on one side in the cementum and on the other in the lamina dura of the alveolar bone (Sharpey's fibers). The oblique direction of these support fibers in their attack on the tooth surface allows the tooth to possess an elasticity enough to distribute the masticatory forces over a large surface of the alveolar process, allowing the tooth to oppose a greater resistance to forces exerted during normal chewing. Two other important components of the periodontal ligament are: cellular elements, consisting of undifferentiated mesenchymal cells and their lines of differentiation in fibroblasts and osteoblasts, together with the neural elements, and vascular tissue fluid from the circulatory system. The periodontal ligament has vessels and cells of the blood system, despite the poor vascularization and innervation characterized by amyelinic nerves appointed to nociceptive perception, and receptors 
associated with proprioception, responsible of the perception of the position of the tooth into alveolus and the spatial relationship between the jaws during mastication [1,2]. PDL plays a relevant role during orthodontic treatment and for this reason it is interesting to characterize its structure and components. In the present manuscript, samples of PDL obtained before any orthodontic treatment have been examined by using different experimental techniques that can offer complementary information on PDL properties. Scanning electron microscopy has been used for investigating PDL structures, while Fourier transform Infrared microspectroscopy ( $\mu$-FT-IR) and Raman microspectroscopy ( $\mu$ - RS) allowed us to characterize PDL from a biochemical point of view.

\section{Materials and Methods}

\subsection{Sample Preparation}

Samples of periodontal ligaments (PDL) of orthodontic patients; aged between 13 and 21 years, and treated with extraction of upper and/or lower premolars, have been selected. Informed consent was obtained from each minor patient's parents or adult patients after providing them detailed information about the clinical trial. The PDL was scarified from radicular surface of the extracted premolars using a one-way lancet. Each sample has dimensions of the order of few $\mathrm{mm}^{3}$. The PDL samples were fixed in $4 \%$ paraformaldehyde (PFH) at least in $3 \mathrm{~h}$. PFH was removed by centrifugation (2000 rpm for $2 \mathrm{~min})$. To fix samples graded series of ethanol solutions $(50 \%-70 \%-$ $80 \%-95 \%$ ) were used. Samples were leaved in each ethanol's solution for $1 \mathrm{~h}$ at room temperature starting from $50 \%$ solution to $95 \%$ and then they were stored in ethanol $100 \%$ until analysis.

\subsection{Experimental Techniques}

\subsubsection{Scanning Electron Microscopy (SEM)}

Collected PDL samples were washed in PBS, fixed in $4 \%$ paraformaldehyde in PBS and then dehydrated with increasing ethanol percentage (30-90\% in water for $5 \mathrm{~min}$, twice $100 \%$ for $15 \mathrm{~min}$ ). After drying in Critical Point Dryer (EMITECH K850), samples were sputter coated with platinumpalladium (Denton Vacuum DESKV, 77 mAmps e 120 sec.) and observed with Supra 40 Zeiss Fieldemission scanning electron microscope (EHT $=5.00 \mathrm{kV}, \mathrm{WD}=22 \mathrm{~mm}$, detector in lens).

\subsubsection{FT-IR Microspectroscopy}

FT-IR spectra were obtained using a Perkin Elmer Spectrum One spectrometer in a transmission geometry using $\mathrm{KBr}$ pellets. Initially, $0.1 \mathrm{~g}$ of a PDL sample was mixed with $5 \mathrm{~mL}$ water and sonicated on ice for $5 \mathrm{~min}$ by using a Soniprep 150 Plus Utrasonic Disgregator. The homogenate was stored at $-20^{\circ} \mathrm{C}$ for $24 \mathrm{~h}$. After this, the sample was lyophilized ( $\left.24 \mathrm{~h}\right)$, using a Lio-5P Freeze Dryer. $\mathrm{KBr}$ pellets were prepared by mixing a small quantity of PDL sample with $\mathrm{KBr}$ (at the ratio of $1 / 100$ ). For every sample two $\mathrm{KBr}$ pellets were prepared. All spectra were obtained using 64 scans in the range from 4000 to $450 \mathrm{~cm}^{-1}$ with a $4 \mathrm{~cm}^{-1}$ spectral resolution. The spectra were preliminarily analyzed using the application routines provided by the software package ("Spectrum" Perkin Elmer Inc., Hopkinton, MA, USA) controlling the whole data acquisition system. The spectra were further analyzed in terms of convoluted peak functions to determine the basic vibrational modes that contribute to the FT-IR signal by using a best-fit peak fitting routine of GRAMS software based on the Levenberg-Marquardt nonlinear least square method. Lorentzian-Gaussian curves were used. Peaks constituting the spectrum were manually selected to define the starting conditions for the best-fit procedure. The bestfit was then performed to determine the optimized intensity, position, and width of the peaks. The performance of the procedure was evaluated by means of the $\chi^{2}$ parameter [3]. 


\subsubsection{Raman Microspectroscopy}

Samples were excited by the light of a He-Ne laser operating at a wavelength $\lambda=633 \mathrm{~nm}$, with a maximum nominal power of $17 \mathrm{~mW}$. The signal was collected by a Jobin-Yvon TriAx 180 monochromator, equipped with a liquid $\mathrm{N}_{2}$ cooled CCD and a grating of 1800 grooves $/ \mathrm{mm}$, allowing a spectral resolution of $4 \mathrm{~cm}^{-1}$. The laser light was focused on the sample surface by means of a $100 \times$ (n.a. $=0.90$ ) optical objective on an excitation area of about $10 \mu \mathrm{m}$ of size. The spectra were obtained using accumulation times ranging in 60-300 s. The spectra were analyzed using the same approach previously described for FT-IR spectra [see also Ref. 4]. For Raman spectra Lorentzian peak shape was used. The band of amide I (located in the 1550- to $1750-\mathrm{cm}^{-1}$ wave number region) was detailly analyzed for obtaining significant information about protein configuration.

\section{Results and Discussion}

\subsection{SEM Observations}

Numerous collagen fibres thickened in bundles are clearly visible at scanning electron microscopy observation as shown in Figure 1A. Figure 2B,C shows in two different area and at different magnification the connection between collagen fibers of periodontal ligament and bone matrix fibres (white asterisk).
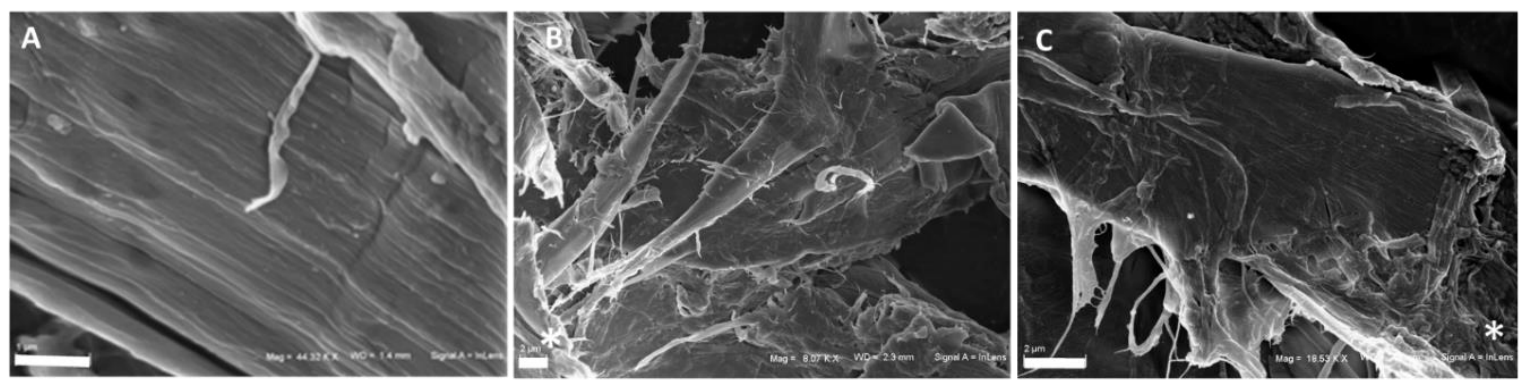

Figure 1. SEM micrographs of periodontal ligaments. Collagen fibers and bone matrix. Scale Bar $=1$ $\mu \mathrm{m}$ in $(\mathbf{A}), 2 \mu \mathrm{m}$ in $(\mathbf{B})$ and $(\mathbf{C})$.

\subsection{FT-IR Results}

In Figure 2 a representative infrared spectrum of PDL is reported together with the results of the deconvolution procedure performed by using Lorentzian-Gaussian curves. The main contribution to the spectrum and the related assignments are reported in Table 1a. The region related to $\mathrm{C}-\mathrm{H}$ bonds in the region 3100-2800 $\mathrm{cm}^{-1}$ can be related to collagen contribution evidenced by SEM micrographs and to a lesser extent to lipid content. In the $1750-1000 \mathrm{~cm}^{-1}$ region, the band at $1656 \mathrm{~cm}^{-1}$ is due to the $\mathrm{C}=\mathrm{O}$ stretching of Amide I. For other peaks see Table 1a. Interesting information about the secondary structure of protein content could be obtained by considering the subcomponents of Amide I and Amide III regions. The deconvolution of Amide I region (1750-1580 $\left.\mathrm{cm}^{-1}\right)$ indicates the presence of different subcomponents that are related to the protein secondary structure. The contributions at $1673 \mathrm{~cm}^{-1}$ is ascribed to $\beta$-turn subcomponent, the ones at 1661 and $1650 \mathrm{~cm}^{-1}$ are related to $\alpha$-helix subcomponent, and the features at 1639 and $1631 \mathrm{~cm}^{-1}$ are due $\beta$-sheets subcomponent. 


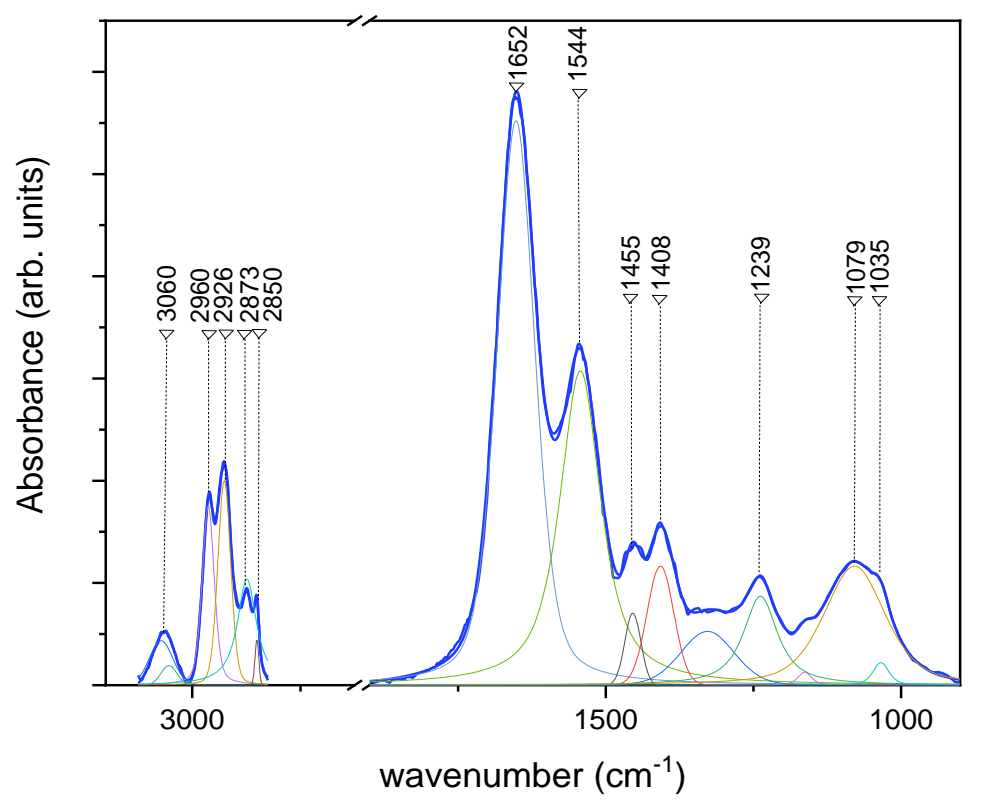

Figure 2. Representative FT-IR spectrum of PDL in the $3100-1000 \mathrm{~cm}^{-1}$ with the deconvolution analysis of peaks with Gaussian- Lorentzian curves (blue line: experimental curve).

\subsection{Raman Microspectroscopy Results}

In Figure 3 a representative Raman spectrum of PDL is reported. As is evident the main contribution due to Amide I and Amide III are evident together with the contributions due to the C$\mathrm{H}$ bonds. These features are related to collagen structure and the bands in the $\mathrm{CH}_{3} / \mathrm{CH}_{2}$ region could be also associated with tissue lipid components. See Ref. [5] and Table $1 \mathrm{~b}$ for other assignments and details.

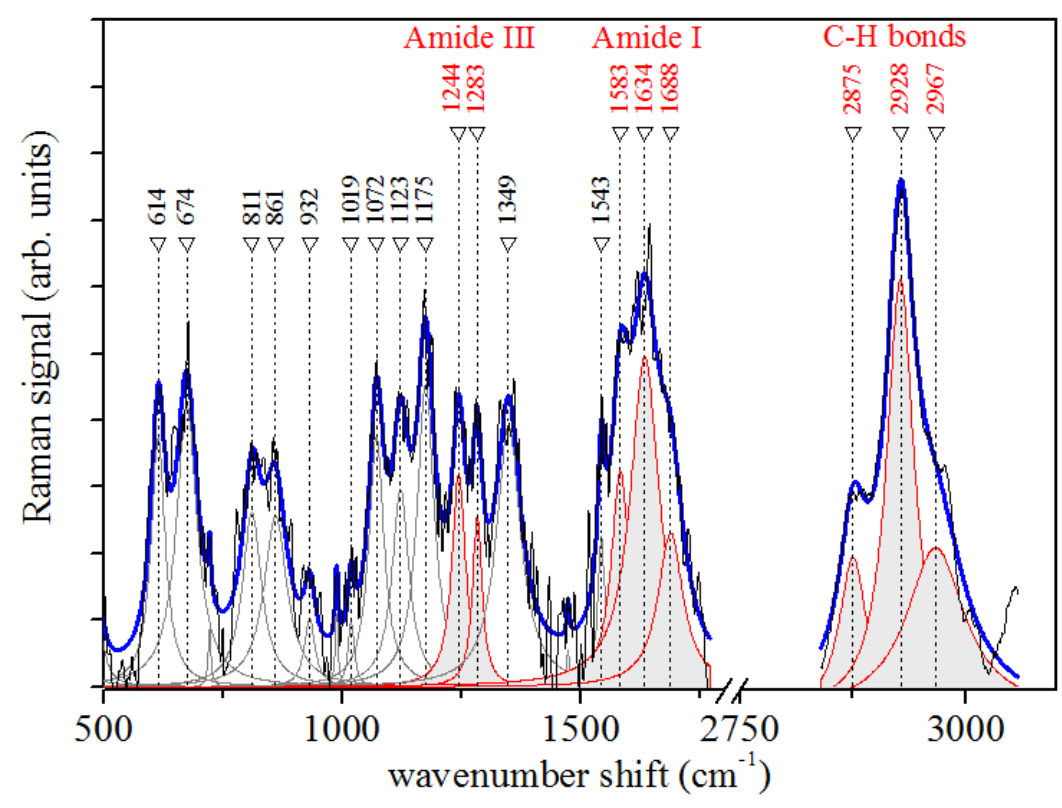

Figure 3. Representative Raman spectrum of PDL. The blue curve is the convolution of the Lorentzian components estimated by fitting the experimental data. 
Also in the case of Raman microspectroscopy, the analysis of subcomponents of Amide I and Amide III has allowed us information about the secondary structures of PDL protein content $[5,6]$.

Table 1. Assignments of the main modes of FT-IR (a) and Raman (b) spectra of PDL according to References reported in $[3,4]$.

(b)

(a)

\begin{tabular}{cc}
\hline $\begin{array}{c}\text { Raman Mode } \\
\boldsymbol{v}\left(\mathbf{c m}^{-1}\right)\end{array}$ & Assignments \\
\hline 614 & phosphate $\delta$ \\
\hline 811 & CC skeleton \\
\hline 861 & proline \\
\hline 932 & phosphate \\
\hline 1019 & C-N proline \\
\hline 1072 & C-N proline \\
\hline 1123 & C-N \\
\hline 1175 & C-O $v$ \\
\hline 1244 & random coil-Amide III \\
\hline 1283 & $\alpha$-helix-Amide III \\
\hline 1349 & CH2 Glycine- \\
& Amide III \\
\hline 1543 & $\mathrm{~N}-\mathrm{H}^{\delta}-$ Amide II \\
\hline 1583 & $\beta$-sheet-Amide I \\
\hline 1634 & $\alpha$-helix-Amide I \\
\hline 1688 & $\beta$-turn-Amide I \\
\hline 2875 & $\mathrm{CH}_{2}$ as. $v$ \\
\hline 2928 & $\mathrm{CH}$ s. $v$ \\
\hline 2967 & $\mathrm{CH}_{3}$ as. $v$ \\
\hline
\end{tabular}

As said before, the Amide I band consisted of major components that are related to the secondary structure of the protein. This band allowed us to obtain information on collagen which is the main PDL component. In particular, the contributions of $\alpha$ helix, $\beta$-sheet, collagen 310-helix and $\beta$-turn subcomponent were centered at about 1642, 1647, 1617 and $1668 \mathrm{~cm}^{-1}$, respectively.

\section{Conclusions}

The reported results highlight the great potential of the techniques used for characterizing human PDL samples from different points of view. In particular, SEM investigation evidences the PDL structure, while $\mu$-FT-IR and $\mu$-RS offer complementary information about biochemical composition of this kind of tissue that plays a very peculiar role during orthodontic treatments when the application of forces can cause structural and biochemical changes.

Author Contributions: Conceptualization, M.L. and L.P.; methodology, C.C., M.P. and M.C.; software, C.C., M.P. and M.C.; investigation, C.C., M.P., M.C. and S.E.; writing-original draft preparation, M.L., C.C. and M.P.; writing-review and editing, M.L., C.C., and M.P. All authors have read and agreed to the published version of the manuscript.

Funding: This research received no external funding

Conflicts of Interest: The authors declare no conflict of interest.

\section{References}


1. Anastasi, G.; Cordasco, G.; Matarese, G.; Rizzo, G.; Nucera, R.; Mazza, M.; Militi, A.; Portelli, M.; Cutroneo, G.; Favaloro, A. An immunohistochemical, histological, and electron-microscopic study of the human periodontal ligament during orthodontic treatment. J. Mol. Med. 2008, 21, 545-554.

2. d'Apuzzo, F.; Cappabianca, S.; Ciavarella, D.; Monsurrò, A.; Silvestrini-Biavati, A.; Perillo, L. Biomarkers of Periodontal tissue remodeling during orthodontic tooth movement in mice and men: Overview and clinical relevance. Sci. World J. 2013, 2013, 105873.

3. Portaccio, M.; d'Apuzzo, F.; Perillo, L.; Grassia, V.; Errico, S.; Lepore, M. Infrared microspectroscopy characterization of gingival crevicular fluid during orthodontic treatment. J. Mol. Struct. 2019, 1176, 847854.

4. Delfino, I.; Perna, G.; Lasalvia, M.; Capozzi, V.; Manti, L.; Camerlingo, C.; Lepore, M. Visible micro-Raman spectroscopy of single human mammary epithelial cells exposed to X-ray radiation. J. Biomed. Optic. 2015, 20, 035003.

5. Perillo, L.; D’Apuzzo, F.; Illario, M.; Laino, L.; Di Spigna, G.; Lepore, M.; Camerlingo, C. Monitoring Biochemical and structural changes in human periodontal ligaments during orthodontic treatment by means of micro-Raman spectroscopy. Sensors 2020, 20, 497, doi:10.3390/s20020497.

6. Camerlingo, C.; d'Apuzzo, F.; Grassia, V.; Perillo, L.; Lepore, M. Micro-Raman spectroscopy for monitoring changes in periodontal ligaments and gingival crevicular fluid. Sensor 2008, 14, 22552-22563.

(C) 2020 by the authors. Submitted for possible open access publication under the terms and conditions of the Creative Commons Attribution (CC BY) license (http://creativecommons.org/licenses/by/4.0/). 